\title{
Pooling of critical, low-utilization resources with unavailability
}

\author{
Loe Schlicher $^{1}$ • Marco Slikker ${ }^{1}$ • Geert-Jan van Houtum ${ }^{1}$
}

Received: 31 May 2016 / Accepted: 23 November 2017 / Published online: 19 December 2017

(C) The Author(s) 2017. This article is an open access publication

\begin{abstract}
We consider an environment in which several independent service providers can collaborate by pooling their critical, low-utilization resources that are subject to unavailability. We examine the allocation of the joint profit for such a pooled situation by studying an associated cooperative game. For this game, we will prove non-emptiness of the core, present a population monotonic allocation scheme and show convexity under some conditions. Moreover, four allocation rules will be introduced and we will investigate whether they satisfy monotonicity to availability, monotonicity to profit, situation symmetry and game symmetry. Finally, we will also investigate whether the payoff vectors resulting from those allocation rules are members of the core.
\end{abstract}

\section{Introduction}

In this paper, we will investigate situations in which several independent service providers keep the same type of critical, low-utilization resources that are subject to unavailability. For instance, one can think of a railway setting with several contractors, each having one tamping machine. Tamping machines are critical resources as they repair unstable, and so unusable, railway tracks. As only a few railway tracks become unstable per year and tamping takes some hours only, the utilization of tamping machines is relatively low. However, tamping machines sometimes fail, are in repair and as a consequence are unavailable for some weeks. One can also think of a setting with several maintenance companies, each having one repairman with specific

$凶$ Loe Schlicher

1.p.j.schlicher@tue.nl

1 School of Industrial Engineering, Eindhoven University of Technology, P.O. Box 513, 5600 MB

Eindhoven, The Netherlands 
knowledge for one and the same type of highly profitable machine. Repairmen are critical resources as they repair those machines. As machines break down only a few times per year and repair takes some hours only, utilization of repairmen is relatively low. However, due to, for example, vacation or illness, repairmen may be unavailable for several weeks. In both examples, it is possible that demand occurs for an unavailable resource. For the railway setting, this leads to an inoperative part of the railway network and as a consequence to high social costs. For the specialized repairmen setting, this leads to down time of the machine which may be very costly as well. As the fraction of time resources are utilized is relative small, and so it is very unlikely that two or more service providers need a resource simultaneously, pooling of resources may be a natural option here. In particular, by pooling of resources, more service can be provided upon request and so additional profit can be realized in the long run. This may encourage the service providers to pool their resources.

We will examine such a pooled situation by considering a stylized model of reality. In this model, we assume that (i) resources switch between being available and unavailable according to underlying stochastic processes, (ii) it does not occur that two or more service providers need a resource simultaneously, (iii) transshipments of resources between service providers occur instantaneously at zero costs and (iv) the profit of each service provider depends non-decreasingly on the long-run fraction of time that at least one resource is available. The first assumption is realistic as there is no reason to assume that unavailability of a resource of a service provider would affect the unavailability of a resource of another service provider. The second assumption is a good approximation of reality when the fraction of time resources are utilized is relative small, which implies that it is unlikely that two or more service providers need a resource simultaneously. The third assumption is realistic in settings wherein service providers are located close (enough) to each other or wherein substantial time is needed to prepare the environment for service. For the latter one, the Dutch railway setting is a good example as it already may take hours to create a safe work environment for tamping. Transshipment costs can be neglected as they are usually small relative to costs for unavailability. The last assumption is realistic in settings wherein service providers operate for a sufficient long time. In this paper, we analyse general non-decreasing profit functions as well as linear profit function, specifically. Nonlinear profit functions fit well in situations, such as the Dutch railway setting, wherein service providers operate based on performance based contracts that reward service providers more as availability increases. Linear profit functions fit well for situations with highly profitable machines for which downtime costs increase proportional to the unavailability of the machines. Under these four assumptions, the model relies on two components only: the long-run fraction of time that at least one resource is available and the profit functions of the service providers.

Although pooling of resources may generate additional profit, it is unclear yet how to allocate this amongst the participating service providers. The use of cooperative game theory can be helpful in here. In particular, we will introduce a cooperative game in order to examine the allocation of the additional profit for such a pooled situation. For this cooperative game, which we will call an availability game, we will contribute in the following way. We will show that there always exists an allocation of the joint profit that cannot be improved upon by any subgroup of players, i.e. 
any coalition. In this case, the so-called core of the associated game is non-empty. Moreover, we present an allocation of the joint profit for every possible coalition such that each player's payoff increases as the coalition to which the player belongs grows larger, i.e. we present a population monotonic allocation scheme. In addition, we will present conditions that ensure that each player's marginal contribution increases as the coalition to which he or she belongs grows larger, i.e. convexity of the associated game. We will also introduce four different allocation rules and investigate whether the payoff vectors resulting from those allocation rules increase for an increasing availability and increasing profit, i.e. satisfy monotonicity to profit and monotonicity to availability. Furthermore, we will investigate whether the payoff vectors resulting from those allocation rules are the same for players that have the same profit function and availabilities, i.e. satisfy situation symmetry, and are the same for players that have the same payoff for every possible coalition, i.e. satisfy game symmetry. Finally, we will also investigate whether the payoff vectors resulting from those allocation rules are members of the core.

This paper can be positioned at the interface of cooperative game theory and operations research problems. In the literature, this research area is summarized under the heading of operations research (OR) games. An overview of OR games can be found in Borm et al. (2001). They divide OR games in five categories, namely connection, routing, scheduling, production and inventory. Availability games are mostly overlapping with the last category. Recent publications in this category focus on economic order quantity situations (Meca et al. 2004), economic lot sizing situations (Van Heuvel et al. 2007; Drechsel and Kimms 2011), newsvendor situations (Özen et al. 2008), truckload delivery situations (Hezarkhani et al. 2016; Li et al. 2016) and spare parts situations (Karsten et al. 2012; Karsten and Basten 2014; Karsten et al. 2015). Recently, Bachrach et al. (2011, 2012, 2013) introduced and investigated a new class of operations research games, called cooperative reliability games, which comes closer to our work. Those games consider a directed network with one sink and one source, where each link is controlled by a self-interested agent. Those links are subject to failures with some fixed probability. The agents can form coalitions to obtain connectivity from the sink to the target node. A fixed reward, which is equal to the probability of achieving connectivity for that coalition, should then be divided amongst the participating agents. In particular, Bachrach et al. (2011) focused on how to approximate the Shapley value for large networks and Bachrach et al. $(2012,2013)$ focused on when cooperative reliability games are convex and balanced. The key difference with their work is that Bachrach et al. $(2011,2012,2013)$ assumed that the reward obtained per coalition depends on a single societal profit function only, while in our model it is assumed that the reward obtained per coalition depends on the sum of the profit functions of all players of that coalition. So, results of Bachrach et al. (2011, 2012, 2013) are not applicable here.

The remainder of this paper is as follows. We start in Sect. 2 with preliminaries on cooperative game theory. In Sect. 3, we introduce cooperative availability games and show general properties regarding those games. In Sect. 4, we introduce four different allocation rules and investigate them on several properties. Finally, we draw conclusions and suggest directions for future research in Sect. 5. 


\section{Preliminaries on cooperative game theory}

In this section, we provide some basic elements of cooperative game theory. Consider a finite set $N$ of players and a function $v: 2^{N} \rightarrow \mathbb{R}$ called the characteristic function, with $v(\emptyset)=0$. The pair $(N, v)$ is called a cooperative game with transferable utility, shortly called a game. A subset $M \subseteq N$ is a coalition and $v(M)$ is the worth coalition $M$ can achieve by itself. The worth can be transferred freely amongst the players. The set $N$ is called the grand coalition. For a coalition $M \subseteq N$, the subgame $\left(M, v_{M}\right)$ is the game with player set $M$ and characteristic function $v_{M}$ with $v_{M}(K)=v(K)$ for all $K \subseteq M$. A game $(N, v)$ is monotonic if the value of every coalition is at least the value of any of its subcoalitions, i.e. $v(M) \leq v(K)$ for all $M, K \subseteq N$ with $M \subseteq K$. When the value of the union of any two disjoint coalitions is larger than or equal to the sum of the values of these disjoint coalitions, a game $(N, v)$ is superadditive, i.e. $v(M)+v(K) \leq v(M \cup K)$ for all $M, K \subseteq N$ with $M \cap K=\emptyset$. A game $(N, v)$ is convex if the marginal contribution of any player to any coalition is less than his marginal contribution to a larger coalition, i.e. $v(K \cup\{i\})-v(K) \geq v(M \cup\{i\})-v(M)$ for all $M \subseteq K \subseteq N \backslash\{i\}$ and all $i \in N$.

An allocation for a game $(N, v)$ is an $N$-dimensional vector $x \in \mathbb{R}^{N}$ describing the payoffs to the players, where player $i \in N$ receives $x_{i}$. An allocation is called efficient if $\sum_{i \in N} x_{i}=v(N)$. This implies that all worth is divided amongst the players of the grand coalition $N$. An allocation is individual rational if $x_{i} \geq v(\{i\})$ for all $i \in N$ and stable if no group of players has an incentive to leave the grand coalition $N$, i.e. $\sum_{i \in M} x_{i} \geq v(M)$ for all $M \subseteq N$. The set of efficient and individual rational allocations, called the imputation set of $(N, v)$, is denoted by

$$
\mathscr{I}(N, v):=\left\{x \in \mathbb{R}^{N} \mid x_{i} \geq v(\{i\}) \text { for all } i \in N \text { and } \sum_{i \in N} x_{i}=v(N)\right\} .
$$

The set of efficient and stable allocations, called the core of $(N, v)$, is denoted by

$$
\mathscr{C}(N, v):=\left\{x \in \mathbb{R}^{N} \mid \sum_{i \in M} x_{i} \geq v(M) \text { for all } M \subseteq N \text { and } \sum_{i \in N} x_{i}=v(N)\right\} .
$$

Following Bondareva (1963) and Shapley (1967), a game $(N, v)$ is called balanced if the core is non-empty. If for every $M \subseteq N$, the corresponding subgame $\left(M, v_{M}\right)$ is balanced, the game is called totally balanced.

A well-known rule that associates an allocation with each game is the Shapley value, proposed by Shapley (1953). This allocation rule can be described in several ways. One is to calculate a weighted average over all marginal contributions that a player can make to any possible coalition. Formally, for any game $(N, v)$ the Shapley value is defined by

$$
\Phi_{i}(N, v)=\sum_{M \subseteq N \backslash\{i\}} \frac{|M| !(|N|-1-|M|) !}{|N| !}(v(M \cup\{i\})-v(M)) \quad \text { for all } i \in N
$$


For any game $(N, v)$, an allocation scheme $y=\left(y_{i, M}\right)_{M \subseteq N, i \in M}$ specifies how to allocate the worth of every coalition. A population monotonic allocation scheme (PMAS), introduced by Sprumont (1990), is an allocation scheme $\left(y_{i, M}\right)_{M \subseteq N, i \in M}$ that is efficient, i.e. $\sum_{i \in M} y_{i, M}=v(M)$ for all $M \subseteq N$, and monotonic, i.e. $y_{i, M} \leq y_{i, K}$ for all $M, K \subseteq N$ with $M \subseteq K$ and all $i \in M$. If a game $(N, v)$ admits a PMAS $y$, then it is totally balanced and its allocation for the grand coalition, $\left(y_{i, N}\right)_{i \in N}$, is a member of the core.

\section{Model description}

In this section, we introduce our model of the underlying situation, which we call an availability situation. Moreover, we define the cooperative game to which we will refer as the associated availability game.

\subsection{Availability situations}

Consider an environment with $N$ a finite set of independent service providers, each providing the same service with a single interchangeable resource (for example a tamping machine or a repairman). For each service provider $i \in N$, we assume that its resource can switch between two states, namely available and unavailable, according to an underlying stochastic process, in which $A_{i} \in(0,1)$ represents the resulting long-run fraction of time that the resource of player $i$ is available.

Remark 1 An example of such a stochastic process is the one in which the resource of any service provider $i \in N$ starts in state available for a fixed time unit, for example a day or an hour, and then, at the end of each time unit in which the resource is available, switches to state unavailable with probability $p_{i}^{a} \in(0,1)$ or remains in the same state with probability $1-p_{i}^{a}$ and, at the end of each time unit in which the resource is unavailable, switches to state available with probability $p_{i}^{u} \in(0,1)$ or remains in the same state with probability $1-p_{i}^{u}$. For such a (discrete time) stochastic process, one can determine easily that the steady-state probability of being available is $\frac{p_{i}^{u}}{p_{i}^{a}+p_{i}^{u}}$ and it holds, with probability 1 , that the long-run fraction of time that the resource is available converges to this steady-state probability. Here, with $Z_{i}^{T}$ the number of time units up to and including time unit $T$ for which the resource is available, the long-run fraction of time that the resource is available is defined as $\lim _{T \rightarrow \infty} Z_{i}^{T} / T$.

From now on, we refer to $A_{i}$ as the availability and to $1-A_{i}$ as the unavailability. We assume that the profit function of each service provider depends on its availability. Let $P_{i}:[0,1] \rightarrow \mathbb{R}_{+}$be this non-decreasing profit function. So, for availability $A_{i}$ service provider $i \in N$ receives a profit of $P_{i}\left(A_{i}\right)$. We assume that there exists a $j \in N$ for which $P_{j}(1)-P_{j}\left(A_{j}\right)>0$. To analyse this setting, we define an availability situation as a tuple $(N, A, P)$ with $N, A=\left(A_{i}\right)_{i \in N}$, and $P=\left(P_{i}\right)_{i \in N}$ as described above. We use $\theta$ to refer to an availability situation $\theta=(N, A, P)$ and $\theta^{\prime}$ to refer to availability situation $\theta^{\prime}=\left(N^{\prime}, A^{\prime}, P^{\prime}\right)$. The set of availability situations is denoted by $\Theta$. 


\subsection{Availability games}

The service providers, from now on called players, can protect against unavailability by pooling their resources. We assume that transshipments of resources between two or multiple players occur instantaneously at zero costs. Moreover, we assume that no two or more players will demand for a resource simultaneously, and so, the new availability of player $i$ as part of coalition $M$ becomes the long-run fraction of time that at least one resource is available. We define this long-run fraction of time that at least one resource of coalition $M$ is available for any player $i \in M$ as follows

$$
A_{i}^{M}=1-\prod_{j \in M}\left(1-A_{j}\right) .
$$

Remark 2 For example, for multiple stochastic processes like the one presented in Remark 1, the relationship in (1) holds with probability 1 , whenever the probabilities of the different players are independent. This follows as a similar relationship holds for the steady-state probabilities. Note there may exist several other (types of) stochastic processes for which (1) holds.

As profit depends on this availability (only), the profit of player $i$ as part of coalition $M$ becomes $P_{i}\left(A_{i}^{M}\right)$, and thus, the profit of coalition $M$ becomes $\sum_{i \in M} P_{i}\left(A_{i}^{M}\right)$. Now, we can define a game corresponding to an availability situation $\theta$.

Definition 1 For any availability situation $\theta=(N, A, P)$, the game $\left(N, v^{\theta}\right)$ with

$$
v^{\theta}(M)=\sum_{i \in M} P_{i}\left(A_{i}^{M}\right)
$$

for all $M \in 2^{N} \backslash\{\emptyset\}$ and $v^{\theta}(\emptyset)=0$ is called the associated availability game.

Example 1 Consider availability situation $\theta \in \Theta$ with $N=\{1,2,3\}, A_{1}=0.6, A_{2}=$ $0.9, A_{3}=0.5, P_{1}(x)=x, P_{2}(x)=2 x$ and $P_{3}(x)=7 x$. In Table 1 , the related availabilities and corresponding profits for $\left(N, v^{\theta}\right)$ are presented per coalition.

\subsection{General properties}

In this section, we will present general properties for availability games. The following two lemma's will be used frequently. The first lemma states that the availability of a coalition is at least the availability of any of its subcoalitions, while the second lemma states that the profit of a coalition is at least the profit of any of its subcoalitions.

Table 1 Corresponding availabilities and profits

\begin{tabular}{lcccccccc}
\hline$M$ & $\emptyset$ & $\{1\}$ & $\{2\}$ & $\{3\}$ & $\{1,2\}$ & $\{1,3\}$ & $\{2,3\}$ & $\{1,2,3\}$ \\
\hline$A_{i}^{M}$ & - & 0.60 & 0.90 & 0.50 & 0.96 & 0.80 & 0.95 & 0.98 \\
$v^{\theta}(M)$ & 0 & 0.60 & 1.80 & 3.50 & 2.88 & 6.40 & 8.55 & 9.80 \\
\hline
\end{tabular}


Lemma 1 For every availability situation $\theta \in \Theta$, it holds that for any $M, K \subseteq N$ with $M \subseteq K$

$$
\prod_{i \in M}\left(1-A_{i}\right) \geq \prod_{i \in K}\left(1-A_{i}\right)
$$

Proof Let $\theta \in \Theta$ be an availability situation and $M, K \subseteq N$ with $M \subseteq K$. We have $0 \leq 1-A_{i} \leq 1$ for all $i \in N$ and consequently

$$
\prod_{i \in M}\left(1-A_{i}\right) \geq \prod_{i \in M}\left(1-A_{i}\right) \cdot \prod_{i \in K \backslash M}\left(1-A_{i}\right)=\prod_{i \in K}\left(1-A_{i}\right),
$$

where the inequality uses $0 \leq \prod_{i \in M}\left(1-A_{i}\right) \leq 1$ for all $M \subseteq N$.

Lemma 2 For every availability situation $\theta \in \Theta$ with $M, K \subseteq N, M \subseteq K$ and $i \in M$, it holds that

$$
P_{i}\left(A_{i}^{M}\right) \leq P_{i}\left(A_{i}^{K}\right)
$$

Proof Let $\theta \in \Theta$ be an availability situation. Then

$$
P_{i}\left(A_{i}^{M}\right)=P_{i}\left(1-\prod_{j \in M}\left(1-A_{j}\right)\right) \leq P_{i}\left(1-\prod_{j \in K}\left(1-A_{j}\right)\right)=P_{i}\left(A_{i}^{K}\right)
$$

where the inequality is a result of (i) Lemma 1 and (ii) the non-decreasing property of $P_{i}$. The first and last equality follow from (1).

As a result of Lemma 2, we can now claim that availability games are monotonic: the value of a coalition is at least the value of any of its subcoalitions.

Proposition 1 Every availability game $\left(N, v^{\theta}\right)$ is monotonic.

Proof Let $\theta \in \Theta$ be an availability situation and $\left(N, v^{\theta}\right)$ be the corresponding availability game. Now, let $M, K \subseteq N$ with $M \subseteq K$. Then

$$
v^{\theta}(M)=\sum_{i \in M} P_{i}\left(A_{i}^{M}\right) \leq \sum_{i \in M} P_{i}\left(A_{i}^{K}\right) \leq \sum_{i \in K} P_{i}\left(A_{i}^{K}\right)=v^{\theta}(K) .
$$

The first and last equality hold by definition. The first inequality holds by Lemma 2 and the second one holds as $P_{i}(x) \in \mathbb{R}_{+}$for all $x \in[0,1]$.

In addition, we are able to show that every availability game $\left(N, v^{\theta}\right)$ is superadditive: the value of the union of disjoint coalitions is larger than or equal to the sum of the values of these coalitions. 
Proposition 2 Every availability game $\left(N, v^{\theta}\right)$ is superadditive.

Proof Let $\theta \in \Theta$ be an availability situation and $\left(N, v^{\theta}\right)$ be the corresponding availability game. Let $M, K \subseteq N$ with $M \cap K=\emptyset$. Then

$$
\begin{aligned}
v^{\theta}(M)+v^{\theta}(K) & =\sum_{i \in M} P_{i}\left(A_{i}^{M}\right)+\sum_{i \in K} P_{i}\left(A_{i}^{K}\right) \\
& \leq \sum_{i \in M} P_{i}\left(A_{i}^{M \cup K}\right)+\sum_{i \in K} P_{i}\left(A_{i}^{M \cup K}\right) \\
& =\sum_{i \in M \cup K} P_{i}\left(A_{i}^{M \cup K}\right)=v^{\theta}(M \cup K) .
\end{aligned}
$$

where the inequality holds by Lemma 2 .

Superadditivity does not suffice to conclude that there always exists an allocation that divides total profit completely such that it cannot be improved upon by any coalition, i.e. the core is non-empty. Following Shapley (1953), convexity of a game is a sufficient condition for core non-emptiness. A game is convex if each player's marginal contribution increases as the coalition to which he or she belongs grows larger. The next example shows that availability games are not convex in general.

Example 2 Consider the situation of Example 1. Observe that $v(\{1,2,3\})-v(\{2,3\})$ $=9.80-8.55=1.25<2.90=6.40-3.50=v(\{1,3\})-v(\{3\})$ and we can conclude that the game is not convex.

Despite that availability games are not convex in general, non-emptiness of the core can still be proved.

Theorem 1 Every availability game $\left(N, v^{\theta}\right)$ has a non-empty core.

Proof Let $\theta \in \Theta$ be an availability situation and $\left(N, v^{\theta}\right)$ be the corresponding availability game. Let $\left(x_{i}\right)_{i \in N}$ be the allocation with

$$
x_{i}=P_{i}\left(A_{i}^{N}\right) \text { for all } i \in N
$$

First, observe that

$$
\sum_{i \in N} x_{i}=\sum_{i \in N} P_{i}\left(A_{i}^{N}\right)=v^{\theta}(N),
$$

and thus, the allocation is efficient. Secondly, observe that for any $M \subseteq N$

$$
\sum_{i \in M} x_{i}=\sum_{i \in M} P_{i}\left(A_{i}^{N}\right) \geq \sum_{i \in M} P_{i}\left(A_{i}^{M}\right)=v^{\theta}(M)
$$

where the inequality holds by Lemma 2 . Given that $\sum_{i \in M} x_{i} \geq v^{\theta}(M)$, the allocation is stable as well. Hence, $\left(x_{i}\right)_{i \in N}$ is an efficient and stable allocation and thus always a member of the core. The core is non-empty. 
We can also claim that availability games have a population monotonic allocation scheme (PMAS): there exists an allocation of the joint profit for every possible coalition such that each player's payoff increases as the coalition to which the player belongs grows larger.

Theorem 2 For every availability situation $\theta \in \Theta$ allocation scheme $\left(a_{i, M}\right)_{M \subseteq N, i \in M}$, given by

$$
a_{i, M}=P_{i}\left(A_{i}^{M}\right) \text { for all } i \in M \text { and all } M \subseteq N
$$

is a population monotonic allocation scheme (PMAS) for $\left(N, v^{\theta}\right)$.

Proof Let $\theta \in \Theta$ be an availability situation. Then, observe that

$$
\sum_{i \in M} a_{i, M}=\sum_{i \in M} P_{i}\left(A_{i}^{M}\right)=v^{\theta}(M)
$$

for all $M \subseteq N$. Secondly, observe that for any $M, K \subseteq N$ with $M \subseteq K$ and $i \in M$ we have

$$
a_{i, M}=P_{i}\left(A_{i}^{M}\right) \leq P_{i}\left(A_{i}^{K}\right)=a_{i, K}
$$

and so $\left(a_{i, M}\right)_{i \in M, M \subseteq N}$ is a PMAS.

Following Sprumont (1990), every game with a PMAS is totally balanced. A game is totally balanced if all of its subgames have non-empty cores. Since every availability game has a PMAS, it is totally balanced as well.

Corollary 1 Every availability game $\left(N, v^{\theta}\right)$ is totally balanced.

In Example 2, it is illustrated that availability games are not convex in general. However, it is of interest to investigate if there exist necessary and sufficient conditions for a class of availability situations for which convexity can be ensured. We will investigate the class of availability situations with linear profit functions, i.e. the class of availability situations for which for every player $i \in N$, there exists a $p_{i} \in \mathbb{R}_{+}$such that $P_{i}(x)=p_{i} x$ for all $x \in[0,1]$. These situations will be called linear availability situations. ${ }^{1}$ The set of linear availability situations will be denoted by $\Theta^{L}$.

Definition 2 Let $\theta \in \Theta^{L}$ be a linear availability situation. Then function $\mathscr{L}_{i j}(\theta)$ is defined by

$$
\mathscr{L}_{i j}(\theta)=A_{j}\left(\sum_{k \in N} p_{k} A_{i}-p_{i}\right)-p_{j} A_{i} \quad \text { for all } i, j \in N \text { with } i \neq j
$$

\footnotetext{
1 Availability games originating from linear availability situations with $p_{j}>0$ for some $j \in N$ and $p_{i}=0$ for all $i \in N \backslash\{j\}$ may be recognized as big boss games (see Muto et al. 1988). As a consequence, availability games originating from linear availability situation may be recognized as linear combinations of big boss games. This does not hold for availability games in general.
} 
Theorem 3 For every linear availability situation $\theta \in \Theta^{L}$ with $|N| \geq 2$, the corresponding game $\left(N, v^{\theta}\right)$ is convex if and only if

$$
\mathscr{L}_{i j}(\theta) \leq 0 \quad \text { for all } i, j \in N \text { with } i \neq j .
$$

Proof See Appendix ${ }^{2}$.

Example 3 Consider the (linear) availability situation of Example 1. Note that $p_{1}=$ $1, p_{2}=2$ and $p_{3}=7$. Then, $\mathscr{L}_{12}(\theta)$ is given by

$$
\mathscr{L}_{12}(\theta)=0.9 \cdot(10 \cdot 0.6-1)-2 \cdot 0.6=3.3>0 .
$$

As derived directly in Example 2, the game is indeed not convex.

For linear availability situations $\theta \in \Theta^{L}$ with $p_{i}=\bar{p} \in \mathbb{R}_{+}$for all $i \in N$, Theorem 3 reduces to an easier result.

Corollary 2 For every linear availability situation $\theta \in \Theta^{L}$ with $N=\{1,2, \ldots, n\}$ with $n \geq 2, p_{i}=\bar{p} \in \mathbb{R}_{+}$for all $i \in N$, and $A_{1} \geq A_{2} \geq \cdots \geq A_{n}$, the corresponding availability game $\left(N, v^{\theta}\right)$ is convex if and only if

$$
|N| A_{1} A_{2}-A_{1}-A_{2} \leq 0 .
$$

Proof See Appendix.

Corollary 2 states that, under specific conditions, the corresponding availability game is convex. For example, availability games with only few players are more likely to be convex than games with many players (under the same highest and second highest availabilities). This may be due to the following effect. The additional profit player $i \in N$ generates when another player $j \in N \backslash\{i\}$ enters the coalition decreases by the size of the coalition player $i \in N$ belongs to. This effect may occur for linear availability situations $\theta \in \Theta^{L}$ where availabilities (and profits) are constant as well.

Corollary 3 For every linear availability situation $\theta \in \Theta^{L}$ with $N=\{1,2, \ldots, n\}$ with $n \geq 2, A_{i}=\bar{A}$ for all $i \in N$, and $p_{1} \leq p_{2} \leq \cdots \leq p_{n}$, the corresponding availability game $\left(N, v^{\theta}\right)$ is convex if and only if

$$
\bar{A} \leq \frac{p_{1}+p_{2}}{\sum_{i \in N} p_{i}} .
$$

Proof See Appendix.

Corollary 4 For every linear availability situation $\theta \in \Theta^{L}$ with $N=\{1,2, \ldots, n\}$ with $n \geq 2, p_{i}=\bar{p} \in \mathbb{R}_{+}$, and $A_{i}=\bar{A}$ for all $i \in N$, the corresponding availability game $\left(N, v^{\theta}\right)$ is convex if and only if

$$
\bar{A} \leq \frac{2}{|N|} .
$$

$\overline{{ }^{2} \text { For the sake of readability, lengthy proofs are presented in the appendix. }}$ 
Proof See Appendix.

Corollaries 2, 3 and 4 can be used to investigate quickly whether games are convex and as a consequence to conclude whether the core is non-empty.

\section{Allocation rules}

In the proof of Theorem 1, an interesting allocation per availability situation, which can be seen as an allocation rule, is presented. Despite that the payoff vector resulting from this allocation rule is a core member of every availability situation, it will not necessarily satisfy any other (appealing) property. Even stronger, there may exist other allocation rules that (i) allocate total profit based on other criteria, (ii) satisfy interesting properties and (iii) have a payoff vector that is a core member for every availability situation as well. For that reason, we will introduce three other (interesting) allocation rules for availability situations. For the, in total, four allocation rules, we will investigate if they satisfy monotonicity to availability, monotonicity to profit, situation symmetry and game symmetry. Finally, we will also investigate the core membership of the payoff vectors resulting from the allocation rules.

\subsection{Four allocation rules}

First, we will formally introduce an allocation rule defined on availability situations.

Definition 3 An allocation rule on availability situations is defined as a mapping $\gamma$ that assigns to any availability situation $\theta \in \Theta$ a vector $\gamma(\theta) \in \mathbb{R}^{N}$.

We will only pay attention to allocation rules that divide the total profit, i.e. $\sum_{i \in N} \gamma_{i}(\theta)=v^{\theta}(N)$ for any availability situation $\theta \in \Theta$. The total profit that can be generated only depends on (i) the availabilities and (ii) the profit functions of the different players. In what follows, we will first introduce three intuitive allocation rules, each depending on the availabilities and profit functions of the different players of the corresponding availability situation. Thereafter, we will present the fourth allocation rule which is based on a well-known allocation rule for cooperative games, namely the Shapley value.

The first allocation rule (which is introduced in the proof of Theorem 1 as an allocation for every availability situation) will allocate to every player the profit, he or she generates with its own profit function while being part of the grand coalition. It is based on the idea that a player that generates more profit than another player under the same availability should also be rewarded more. This allocation rule, which we call Own Profit (OP), is described for any availability situation $\theta \in \Theta$ by

$$
\mathrm{OP}_{i}(\theta)=P_{i}\left(A_{i}^{N}\right) \quad \text { for all } i \in N
$$

A possible drawback of the first allocation rule is that players are not rewarded directly for the impact of their own availability (on the profit functions of others). The second allocation rule overcomes this by allocating the total profit proportional 
to the availabilities of the players. The idea behind this allocation rule is that the more a player is available, the more it can help others, and for this, it will be rewarded. Formally, for every availability situation $\theta \in \Theta$, this allocation rule, which we call Proportional to Availability (PA), is defined by

$$
\operatorname{PA}_{i}(\theta)=\frac{A_{i}}{\sum_{j \in N} A_{j}} v^{\theta}(N) \quad \text { for all } i \in N
$$

A possible drawback of the second allocation rule is that players are not rewarded directly for the profit generated with their own profit function while being part of the grand coalition. The third allocation rule will not overcome this (nor the other) drawback. However, it tries to find another intuitive way of dividing the profit based on the availabilities and profit functions. This allocation rule will first allocate the individual profit, i.e. the profit that every player would obtain in the individual situation, to every player. In fact, every player will be rewarded for their own availability and profit function. Then, the remaining part of the total profit, i.e. the surplus, will be divided proportional to the players' profit loss due to unavailability. We measure the profit loss due to unavailability as the difference in profit a player can bring in with $100 \%$ availability and with their (own) individual availability. The idea behind this part is that players are rewarded for their profit potential they can bring with $100 \%$ availability. Formally, for every availability situation $\theta \in \Theta$ this allocation rule, which we call Surplus Proportional to profit Potential (SPP), is defined by ${ }^{3}$

$\operatorname{SPP}_{i}(\theta)=v^{\theta}(\{i\})+\frac{P_{i}(1)-P_{i}\left(A_{i}\right)}{\sum_{j \in N}\left[P_{j}(1)-P_{j}\left(A_{j}\right)\right]}\left(v^{\theta}(N)-\sum_{j \in N} v^{\theta}(\{j\})\right) \quad$ for all $i \in N$.

The last allocation rule that will be introduced is the Shapley value. The Shapley value (Shapley 1953) is a well-known (and accepted) allocation rule for cooperative games. It is the only one that satisfies the efficiency, monotonicity, symmetry and dummy property simultaneously. We will define the Shapley Value (SV) for every availability situation $\theta \in \Theta$ by

$$
\mathrm{SV}_{i}(\theta)=\Phi_{i}\left(N, v^{\theta}\right) \text { for all } i \in N
$$

\subsection{Properties of allocation rules}

In this section, we will investigate whether the allocation rules satisfy intuitive properties as monotonicity to availability, monotonicity to profit, situation symmetry and game symmetry. Finally, we will also investigate whether the payoff vectors resulting from the allocation rules are core members.

\footnotetext{
3 One can also be interested in the situation wherein the profit loss due to unavailability is with respect to $A^{N}$ (rather than 100\%). Then, allocation rule SSP boils down to OP.
} 


\subsubsection{Monotonicity to availability}

Suppose the availability of a player increases. Then, this specific player is able to generate more profit. Moreover, as the total availability increases, other players can generate more profit as well. Hence, it is natural to assume that players do not expect decreases in their allocations. We will investigate whether the allocation rules will allocate to all players not less when the availability of any player increases, i.e. satisfy monotonicity to availability.

Definition 4 An allocation rule $\gamma$ satisfies monotonicity to availability on $D \subseteq \Theta$ if for any two availability situations $\theta, \theta^{\prime} \in D$, where $\theta$ and $\theta^{\prime}$ coincide except for the availability of player $j$ with $A_{j} \leq A_{j}^{\prime}$

$$
\gamma_{i}(\theta) \leq \gamma_{i}\left(\theta^{\prime}\right) \text { for all } i \in N
$$

The following example will show that allocation rules PA, SPP and SV do not satisfy monotonicity to availability on $\Theta$.

Example 4 Consider availability situation $\theta \in \Theta$ with $N=\{1,2,3\}, A_{1}=0.5$, $A_{2}=0.5, A_{3}=0.5$ and

$$
P_{1}(x)=P_{2}(x)=\left\{\begin{array}{ll}
x & \text { if } 0 \leq x \leq \frac{1}{2} \\
\frac{1}{2} & \text { if } \frac{1}{2}<x<1 \\
1 & \text { if } \quad x=1,
\end{array} \quad P_{3}(x)= \begin{cases}x & \text { if } 0 \leq x \leq \frac{1}{2} \\
1 & \text { if } \frac{1}{2}<x \leq 1\end{cases}\right.
$$

Moreover, consider situation $\theta^{\prime} \in \Theta$, which coincides with $\theta$ except that $A_{3}^{\prime}=0.75$. In Table 2, the four allocations regarding those two situations $\theta$ and $\theta^{\prime}$ are depicted for all three players.

Allocation rules PA, SPP and SV do not satisfy monotonicity to availability, since

$$
\begin{aligned}
\operatorname{PA}_{2}(\theta) & >\operatorname{PA}_{2}\left(\theta^{\prime}\right), \\
\operatorname{SPP}_{2}(\theta) & >\operatorname{SPP}_{2}\left(\theta^{\prime}\right), \\
\operatorname{SV}_{2}(\theta) & >\operatorname{SV}_{2}\left(\theta^{\prime}\right)
\end{aligned}
$$

\begin{tabular}{|c|c|c|c|c|c|c|c|c|c|c|c|}
\hline & $i$ & $\mathrm{OP}_{i}$ & $\mathrm{PA}_{i}$ & $\mathrm{SPP}_{i}$ & $\mathrm{SV}_{i}$ & & $i$ & $\mathrm{OP}_{i}$ & $\mathrm{PA}_{i}$ & $\mathrm{SPP}_{i}$ & $\overline{\mathrm{SV}_{i}}$ \\
\hline \multirow{3}{*}{$\theta$} & 1 & $\frac{1}{2}$ & $\frac{2}{3}$ & $\frac{2}{3}$ & $\frac{7}{12}$ & \multirow{3}{*}{$\theta^{\prime}$} & 1 & $\frac{1}{2}$ & $\frac{4}{7}$ & $\frac{1}{2}$ & $\frac{1}{2}$ \\
\hline & 2 & $\frac{1}{2}$ & $\frac{2}{3}$ & $\frac{2}{3}$ & $\frac{7}{12}$ & & 2 & $\frac{1}{2}$ & $\frac{4}{7}$ & $\frac{1}{2}$ & $\frac{1}{2}$ \\
\hline & 3 & 1 & $\frac{2}{3}$ & $\frac{2}{3}$ & $\frac{10}{12}$ & & 3 & 1 & $\frac{6}{7}$ & 1 & 1 \\
\hline
\end{tabular}

Note that this example can also be constructed with continuous profit functions. $\diamond$

Proposition 3 Allocation rule OP satisfies monotonicity to availability on $\Theta$.

Table 2 Allocations for availability game 
Proof See Appendix.

For linear availability situations, we obtain different results regarding monotonicity to availability. The following example will be used to show that allocation rules PA and SV do not satisfy monotonicity to availability on $\Theta^{L}$.

Example 5 Consider the (linear) availability situation $\theta \in \Theta^{L}$ of Example 1. Moreover, consider situation $\theta^{\prime} \in \Theta^{L}$, which coincides with $\theta$ except that $A_{1}^{\prime}=0.8$ now. In Table 3, the four allocations regarding those two situations $\theta$ and $\theta^{\prime}$ are depicted for all three players. All numbers are rounded to two decimals.

Allocation rules PA and SV do not satisfy monotonicity to availability, since

$$
\begin{aligned}
& \operatorname{PA}_{2}(\theta)>\operatorname{PA}_{2}\left(\theta^{\prime}\right), \\
& \operatorname{SV}_{2}(\theta)>\operatorname{SV}_{2}\left(\theta^{\prime}\right) .
\end{aligned}
$$

Proposition 4 Allocation rules OP and SPP satisfy monotonicity to availability on $\Theta^{L}$.

Proof See Appendix.

\subsubsection{Monotonicity to profit}

Suppose the profit function of a player changes such that the difference between the outcomes of the old and the new profit function is non-decreasing in the argument, i.e. in (total) availability. Then, this specific player is able to generate more profit. Despite that the other players will not generate more profit themselves, they are responsible (in terms of availability) for the (extra) profit of the specific player as well. Hence, it is natural to assume that players do not expect decreases in their allocations. We will investigate whether the allocation rules will allocate to all players not less when the difference between the outcome of the new and old profit function of a specific player is non-decreasing in (total) availability, i.e. satisfy monotonicity to profit.

Definition 5 An allocation rule $\gamma$ satisfies monotonicity to profit on $D \subseteq \Theta$ if for any two availability situations $\theta, \theta^{\prime} \in D$, where $\theta$ and $\theta^{\prime}$ coincide except for the profit of player $j$ with $P_{j}^{\prime}(x)-P_{j}(x)$ non-decreasing in $x$,

$$
\gamma_{i}(\theta) \leq \gamma_{i}\left(\theta^{\prime}\right) \quad \text { for all } i \in N
$$

\begin{tabular}{|c|c|c|c|c|c|c|c|c|c|c|c|}
\hline & $i$ & $\mathrm{OP}_{i}$ & $\mathrm{PA}_{i}$ & $\mathrm{SPP}_{i}$ & $\mathrm{SV}_{i}$ & & $i$ & $\mathrm{OP}_{i}$ & $\mathrm{PA}_{i}$ & $\operatorname{SPP}_{i}$ & $\mathrm{SV}_{i}$ \\
\hline \multirow{3}{*}{$\theta$} & 1 & 0.98 & 2.94 & 0.98 & 1.28 & \multirow{3}{*}{$\theta^{\prime}$} & 1 & 0.99 & 3.60 & 0.99 & 1.52 \\
\hline & 2 & 1.96 & 4.41 & 1.99 & 2.95 & & 2 & 1.98 & 4.05 & 2.00 & 2.70 \\
\hline & 3 & 6.86 & 2.45 & 6.83 & 5.57 & & 3 & 6.93 & 2.25 & 6.91 & 5.68 \\
\hline
\end{tabular}

Table 3 Allocations for availability game 
The following example will show that allocation rule SPP does not satisfy monotonicity to profit on $\Theta^{L}$.

Example 6 Consider an availability situation $\theta \in \Theta^{L}$ with $N=\{1,2,3\}, A_{1}=0.6$, $A_{2}=0.7, A_{3}=0.5, p_{1}=1, p_{2}=3$ and $p_{3}=9$. Moreover, consider situation $\theta^{\prime} \in \Theta^{L}$, which coincides with $\theta$ except that $p_{1}^{\prime}=10$ now. In Table 4 , the four allocations regarding those two situations $\theta$ and $\theta^{\prime}$ are depicted for all three players. Note that all numbers are rounded to two decimals.

Allocation rule SPP does not satisfy monotonicity to profit, since

$$
\operatorname{SPP}_{3}(\theta)>\operatorname{SPP}_{3}\left(\theta^{\prime}\right)
$$

Proposition 5 Allocation rules OP, PA and SV satisfy monotonicity to profit on $\Theta$.

Proof See Appendix.

\subsubsection{Situation symmetry}

Suppose that two players have the same profit function and availability. Then, those players both generate the same profit and both help other players (in terms of availability) in the same way. Hence, it is natural to assume that those players expect the same allocation. We will investigate whether the allocation rules will indeed allocate the same to both players, i.e. satisfy situation symmetry. For this, we will introduce some new definitions.

Definition 6 For any availability situation $\theta \in \Theta$, players $i, j \in N$ with $i \neq j$ are called situation symmetric if

$$
P_{i}(x)=P_{j}(x) \text { for all } x \in[0,1] \text { and } A_{i}=A_{j}
$$

Definition 7 An allocation rule $\gamma$ satisfies situation symmetry on $D \subseteq \Theta$ if for all $\theta \in D$ and all situation symmetric players $i, j \in N$ with $i \neq j$ it holds that

$$
\gamma_{i}(\theta)=\gamma_{j}(\theta)
$$

Proposition 6 Allocation rules OP, PA, SPP and SV satisfy situation symmetry on $\Theta$.

\begin{tabular}{|c|c|c|c|c|c|c|c|c|c|c|c|}
\hline & $i$ & $\mathrm{OP}_{i}$ & $\mathrm{PA}_{i}$ & $\mathrm{SPP}_{i}$ & $\mathrm{SV}_{i}$ & & $i$ & $\mathrm{OP}_{i}$ & $\mathrm{PA}_{i}$ & $\mathrm{SPP}_{i}$ & $\mathrm{SV}_{i}$ \\
\hline \multirow{3}{*}{$\theta$} & 1 & 0.94 & 4.07 & 0.95 & 1.69 & \multirow{3}{*}{$\theta^{\prime}$} & 1 & 9.40 & 6.89 & 9.44 & 8.83 \\
\hline & 2 & 2.82 & 4.75 & 2.88 & 3.54 & & 2 & 2.82 & 8.04 & 2.87 & 4.38 \\
\hline & 3 & 8.46 & 3.39 & 8.39 & 6.98 & & 3 & 8.46 & 5.74 & 8.37 & 7.46 \\
\hline
\end{tabular}

Proof See Appendix.

Table 4 Allocations for availability game 


\subsubsection{Game symmetry}

Suppose that player $i$ and player $j$ have the same individual profit, but not necessarily the same profit functions and availabilities. Moreover, assume that the total profit of any coalition including player $i$ equals the total profit of the same coalition including player $j$ rather than $i$. So, both players are symmetric, but now in terms of the corresponding availability game. Hence, it is natural to assume that both players expect the same allocation. We will investigate whether the allocation rules will allocate the same to both players, i.e. satisfy game symmetry. We will first introduce the definition of symmetric players in terms of availability games.

Definition 8 For any availability situation $\theta \in \Theta$ players $i, j \in N$ with $i \neq j$ are called game symmetric if for the corresponding availability game $\left(N, v^{\theta}\right)$

$$
v^{\theta}(M \cup\{i\})=v^{\theta}(M \cup\{j\}) \text { for all } M \subseteq N \backslash\{i, j\} .
$$

Definition 9 An allocation rule $\gamma$ satisfies game symmetry on $D \subseteq \Theta$ if for all $\theta \in D$ and all game symmetric players $i, j \in N$ with $i \neq j$ it holds that

$$
\gamma_{i}(\theta)=\gamma_{j}(\theta)
$$

The following example will show that allocation rules $O P, P A$ and SPP do not satisfy game symmetry on $\Theta^{L}$.

Example 7 Consider a linear availability situation $\theta \in \Theta^{L}$ with $N=\{1,2,3\}, A_{1}=$ $0.7, A_{2}=0.8, A_{3}=0.9, p_{1}=9, p_{2}=40$ and $p_{3}=7$. Then $v^{\theta}(\{1\})=6.3=$ $v^{\theta}(\{3\})$ and $v^{\theta}(\{1,2\})=40.06=v^{\theta}(\{2,3\})$, and thus, we can conclude that players 1 and 3 are game symmetric. The corresponding allocations are presented in Table 5. All numbers are rounded to two decimals.

The allocation rules OP, PA and SPP do not satisfy game symmetry, since

$$
\begin{aligned}
\mathrm{OP}_{1}(\theta) & >\mathrm{OP}_{3}(\theta), \\
\operatorname{PA}_{1}(\theta) & <\operatorname{PA}_{3}(\theta), \\
\operatorname{SPP}_{1}(\theta) & >\operatorname{SPP}_{3}(\theta) .
\end{aligned}
$$

Proposition 7 Allocation rule SV satisfies game symmetry on $\Theta$.

\begin{tabular}{|c|c|c|c|c|c|}
\hline & $i$ & $\mathrm{OP}_{i}$ & $\mathrm{PA}_{i}$ & $\mathrm{SPP}_{i}$ & $\mathrm{SV}_{i}$ \\
\hline \multirow{3}{*}{$\theta$} & 1 & 8.95 & 16.24 & 8.92 & 9.18 \\
\hline & 2 & 39.76 & 18.55 & 39.76 & 37.30 \\
\hline & 3 & 6.96 & 20.87 & 6.98 & 9.18 \\
\hline
\end{tabular}

Proof Let $\theta \in \Theta$ be an availability situation. Moreover, let $i, j \in N$ with $i \neq j$ be two game symmetric players in $\left(N, v^{\theta}\right)$. Following Shapley (1953), it holds that

Table 5 Allocations for availability game 
$\Phi_{i}\left(N, v^{\theta}\right)=\Phi_{j}\left(N, v^{\theta}\right)$. As a consequence, $\operatorname{SV}_{i}(\theta)=\Phi_{i}\left(N, v^{\theta}\right)=\Phi_{j}\left(N, v^{\theta}\right)=$ $\mathrm{SV}_{j}(\theta)$, which concludes that $\mathrm{SV}$ satisfies game symmetry.

\subsubsection{The core}

In Sect. 3.3, we already investigated the non-emptiness of the core. This result was based on finding a payoff vector that always belongs to the core. Now, we will investigate whether the payoff vectors resulting from the allocation rules are always members of the core as well.

The following example will show that there exists an availability situation $\theta \in \Theta$ for which payoff vectors $\operatorname{PA}(\theta), \operatorname{SPP}(\theta)$ and $\operatorname{SV}(\theta)$ are not core elements.

Example 8 Consider the availability situation of Example 4. Then, the corresponding game $\left(N, v^{\theta}\right)$ is given by $v^{\theta}(\{1\})=v^{\theta}(\{2\})=v^{\theta}(\{3\})=\frac{1}{2}, v^{\theta}(\{1,3\})=$ $v^{\theta}(\{2,3\})=1 \frac{1}{2}, v^{\theta}(\{1,2\})=1$ and $v^{\theta}(\{1,2,3\})=2$. The payoff vectors resulting from allocation rules PA, SPP and SV (see Table 2) are not elements of the core, since

$$
\begin{gathered}
\mathrm{PA}_{1}(\theta)+\mathrm{PA}_{3}(\theta)=\frac{2}{3}+\frac{2}{3}<1 \frac{1}{2}=v^{\theta}(\{1,3\}) \\
\operatorname{SPP}_{1}(\theta)+\operatorname{SPP}_{3}(\theta)=\frac{2}{3}+\frac{2}{3}<1 \frac{1}{2}=v^{\theta}(\{1,3\}) \\
\operatorname{SV}_{1}(\theta)+\operatorname{SV}_{3}(\theta)=\frac{7}{12}+\frac{10}{12}=1 \frac{5}{12}<1 \frac{1}{2}=v^{\theta}(\{1,3\})
\end{gathered}
$$

From the proof of Theorem 1, the following result is obtained immediately.

Corollary 5 For every availability situation $\theta \in \Theta$, it holds that

$$
O P(\theta) \in \mathscr{C}\left(N, v^{\theta}\right)
$$

The following example will show that there exists a linear availability situation $\theta \in \Theta^{L}$ for which payoff vectors $\operatorname{PA}(\theta)$ and $\operatorname{SV}(\theta)$ are not core elements.

Example 9 Consider the (linear) availability situation $\theta \in \Theta^{L}$ of Example 1. The related allocations are presented in Table 6 . All values are rounded to two decimal places.

Table 6 Allocations for availability game

\begin{tabular}{cccccc}
\hline & $i$ & $\mathrm{OP}_{i}$ & $\mathrm{PA}_{i}$ & $\mathrm{SPP}_{i}$ & $\mathrm{SV}_{i}$ \\
\hline \multirow{4}{*}{0} & 1 & 0.98 & 2.94 & 0.98 & 1.28 \\
& 2 & 1.96 & 4.41 & 1.99 & 2.95 \\
& 3 & 6.86 & 2.45 & 6.83 & 5.57 \\
\hline
\end{tabular}


The payoff vectors resulting from allocation rules PA and SV are not elements of the core, since

$$
\begin{aligned}
& \mathrm{PA}_{2}(\theta)+\mathrm{PA}_{3}(\theta)<4.42+2.46=6.88<v^{\theta}(\{2,3\}), \\
& \mathrm{SV}_{2}(\theta)+\mathrm{SV}_{3}(\theta)<2.96+5.58=8.54<v^{\theta}(\{2,3\})
\end{aligned}
$$

Based on Corollary 5 and Example 9, it remains to investigate whether payoff vectors resulting from SPP are core members. We make use of the following lemma.

Lemma 3 Let $\theta \in \Theta^{L}$ be a linear availability situation with $x_{i}=1-A_{i}$ for all $i \in N$. Then for all $M \subseteq N$ it holds that

$$
\sum_{i \in M} p_{i}\left(\prod_{j \in M} x_{j}\right) \geq \frac{\sum_{i \in M} p_{i} x_{i}}{\sum_{j \in N} p_{j} x_{j}} \sum_{i \in N} p_{i}\left(\prod_{j \in N} x_{j}\right) .
$$

Proof See Appendix.

Proposition 8 For every availability situation $\theta \in \Theta^{L}$ it holds that

$$
\operatorname{SPP}(\theta) \in \mathscr{C}\left(N, v^{\theta}\right)
$$

Proof See Appendix.

Following Shapley (1953), the Shapley value is a member of the core if the corresponding game is convex. In Theorem 3, necessary and sufficient conditions are given for convexity of games associated with linear availability situations. Combining them leads to our last result immediately.

Corollary 6 For linear availability situations $\theta \in \Theta^{L}$ with $\mathscr{L}_{i j}(\theta) \leq 0$ for all $i, j \in N$ and $i \neq j, S V(\theta)$ is a member of the core of $\left(N, v^{\theta}\right)$.

\section{Conclusions and future research}

We formulated a stylized model of reality in which several independent service providers can collaborate by pooling their critical, low-utilization resources that are subject to unavailability. We examined the allocation of the joint profit for such a pooled situation by studying an associated cooperative game. For this game, we proved that there always exists an allocation of the joint profit that cannot be improved upon by any coalition. In addition, we showed that there exists an allocation of the joint profit for every possible coalition such that each player's payoff increases as the coalition to which the player belongs grows larger. Moreover, we discussed four allocation rules and investigated whether they satisfy intuitive properties such as monotonicity to availability, monotonicity to profit, situation symmetry and game symmetry. Next to that, we investigated whether the payoff vectors resulting for those allocation rules are core members. In Tables 7 and 8, all results have been summarized. 
With respect to the original underlying real-life examples (of tamping machines and specialized repairmen needed for highly profitable machines), these results provide operational insights. For instance, as (in terms of the game) there always exists an allocation of the joint profit that cannot be improved upon by any coalition, investigating collaboration between several contractors or maintenance companies is a worthwhile endeavour. However, it is likely that intuitive and simply-to-implement allocations rules, such as PA and SPP who divide profit proportionally to criteria such as availability and profit, may have some shortcomings in practice. For instance, it is well possible that the payoff vectors of these allocation rules may not be part of the core as well as that they may lack the monotonicity to availability property, which implies that an increase in the availability not necessarily implies an increase in the profit allocation. A possible drawback of allocation rule SV is its implementation, as it requires calculating the values of all coalitions, which may be problematic as this increases exponentially in the number of players. Oppositely, it seems that allocation rule OP, which allocates the profit, he or she generates with its own profit function while being part of the grand coalition, is a good starting point as it is easy to implement, coreguaranteed and dominates allocation rules PA and SPP in terms of the investigated properties.

For further research, it would be interesting to study the following extensions. First, one could look at an extended model with the property that two or more service providers may demand for a resource simultaneously. In this case, it is not always possible (anymore) that another resource can take over demand. Moreover, we can extend the model by including that (i) the individual resource availability depends on the number of parties that participate in the pool and (ii) exchange costs are included for transporting resources to other parties in case of pooling of the resources.

Table 7 Results for availability situations

\section{$\checkmark$ : Satisfies property $\times$ : Does not (always) satisfy property}

Table 8 Results for linear availability situations

${ }^{a}$ Satisfies property if conditions of Corollary 6 hold

\begin{tabular}{lcccc}
\hline Properties & OP & PA & SPP & SV \\
\hline Monotonicity to availability & $\checkmark$ & $\times$ & $\times$ & $\times$ \\
Monotonicity to profit & $\checkmark$ & $\checkmark$ & $\times$ & $\checkmark$ \\
Situation symmetry & $\checkmark$ & $\checkmark$ & $\checkmark$ & $\checkmark$ \\
Game symmetry & $\times$ & $\times$ & $\times$ & $\checkmark$ \\
Member of the core & $\checkmark$ & $\times$ & $\times$ & $\times$ \\
\hline
\end{tabular}

\begin{tabular}{lcccc}
\hline Properties & OP & PA & SPP & SV \\
\hline Monotonicity to availability & $\checkmark$ & $\times$ & $\checkmark$ & $\times$ \\
Monotonicity to profit & $\checkmark$ & $\checkmark$ & $\times$ & $\checkmark$ \\
Situation symmetry & $\checkmark$ & $\checkmark$ & $\checkmark$ & $\checkmark$ \\
Game symmetry & $\times$ & $\times$ & $\times$ & $\checkmark$ \\
Member of the core & $\checkmark$ & $\times$ & $\checkmark$ & $\times{ }^{\mathrm{a}}$ \\
\hline
\end{tabular}


Acknowledgements We thank the review team for their helpful comments. This article is based upon work supported by the Netherlands Organisation for Scientific Research (NWO) and ProRail (438-12-305).

Open Access This article is distributed under the terms of the Creative Commons Attribution 4.0 International License (http://creativecommons.org/licenses/by/4.0/), which permits unrestricted use, distribution, and reproduction in any medium, provided you give appropriate credit to the original author(s) and the source, provide a link to the Creative Commons license, and indicate if changes were made.

\section{Appendix}

Proof of Theorem 3 Let $\theta \in \Theta^{L}$ be a linear availability situation with $|N| \geq 2$ and $\left(N, v^{\theta}\right)$ be the corresponding availability game. We will show that the corresponding availability game is convex if and only if $\mathscr{L}_{i j}(\theta) \leq 0$ for all $i, j \in N$ with $i \neq j$.

$(\Rightarrow)$ Suppose the availability game is convex, i.e. (Shapley 1953), then

$$
v^{\theta}(M \cup\{i, j\})-v^{\theta}(M \cup\{j\})-\left(v^{\theta}(M \cup\{i\})-v^{\theta}(M)\right) \geq 0
$$

for all $i, j \in N$ with $i \neq j$ and all $M \subseteq N \backslash\{i, j\}$. Let $i, j \in N$ with $i \neq j$ and $M \subseteq N \backslash\{i, j\}$. Based on (4), it holds that

$$
\begin{aligned}
0 \leq & v^{\theta}(M \cup\{i, j\})-v^{\theta}(M \cup\{j\})-\left(v^{\theta}(M \cup\{i\})-v^{\theta}(M)\right) \\
= & \sum_{k \in M \cup\{i, j\}} p_{k}\left(1-\prod_{l \in M \cup\{i, j\}}\left(1-A_{l}\right)\right)-\sum_{k \in M \cup\{j\}} p_{k}\left(1-\prod_{l \in M \cup\{j\}}\left(1-A_{l}\right)\right) \\
& -\sum_{k \in M \cup\{i\}} p_{k}\left(1-\prod_{l \in M \cup\{i\}}\left(1-A_{l}\right)\right)+\sum_{k \in M} p_{k}\left(1-\prod_{l \in M}\left(1-A_{l}\right)\right) \\
= & \sum_{k \in M \cup\{j\}} p_{k}\left(A_{i} \prod_{l \in M \cup\{j\}}\left(1-A_{l}\right)\right)+p_{i}\left(1-\prod_{l \in M \cup\{i, j\}}\left(1-A_{l}\right)\right) \\
& -\sum_{k \in M} p_{k}\left(A_{i} \prod_{l \in M}\left(1-A_{l}\right)\right)-p_{i}\left(1-\prod_{l \in M \cup\{i\}}\left(1-A_{l}\right)\right) \\
= & \prod_{l \in M \cup\{j\}}\left(1-A_{l}\right)\left(\sum_{k \in M \cup\{j\}} p_{k} A_{i}-p_{i}\left(1-A_{i}\right)\right) \\
& -\prod_{l \in M}\left(1-A_{l}\right)\left(\sum_{k \in M} p_{k} A_{i}-p_{i}\left(1-A_{i}\right)\right) \\
= & \prod_{l \in M}\left(1-A_{l}\right)\left(\left(1-A_{j}\right)\left(\sum_{k \in M \cup\{i, j\}} p_{k} A_{i}-p_{i}\right)-\left(\sum_{k \in M \cup\{i\}} p_{k} A_{i}-p_{i}\right)\right),
\end{aligned}
$$


where the first equality follows by definition. The second equality follows by combining all terms $k \in M \cup\{j\}$ from the first and second summation into one summation and combining all terms $k \in M$ from the third and fourth summation into one summation. In the two new summations, we combine the product terms and use that $A_{i}=1-\left(1-A_{i}\right)$. Finally, we write down the terms that are left from the original summations. In the third equality, the product term $\prod_{l \in M \cup j}\left(1-A_{l}\right)$ is taken out of the first and second term and the product term $\prod_{l \in M}\left(1-A_{l}\right)$ is taken out of the third and fourth term. Moreover, $p_{i} \cdot 1$ and $-p_{i} \cdot 1$ cancel out against each other. In the fourth equality, the product term $\prod_{l \in M}\left(1-A_{l}\right)$ is taken out of the whole equality and $-p_{i}\left(1-A_{i}\right)$ is written as $p_{i} A_{i}-p_{i}$, where $p_{i} A_{i}$ is finally included in the summation.

As $A_{i} \in(0,1)$ for all $i \in N$, it holds that $\prod_{l \in M}\left(1-A_{l}\right)>0$. If the last expression is divided by $\prod_{l \in M}\left(1-A_{l}\right)$, we obtain

$$
\begin{aligned}
0 & \leq\left(1-A_{j}\right)\left(\sum_{k \in M \cup\{i, j\}} p_{k} A_{i}-p_{i}\right)-\left(\sum_{k \in M \cup\{i\}} p_{k} A_{i}-p_{i}\right) \\
& =p_{j} A_{i}-A_{j}\left(\sum_{k \in M \cup\{i, j\}} p_{k} A_{i}-p_{i}\right) .
\end{aligned}
$$

This is equivalent to

$$
A_{j}\left(\sum_{k \in M \cup\{i, j\}} p_{k} A_{i}-p_{i}\right)-p_{j} A_{i} \leq 0 .
$$

As $i, j \in N$ with $i \neq j$ and $M \subseteq N \backslash\{i, j\}$ were chosen arbitrarily, (5) holds for any $i, j \in N$ with $i \neq j$ and all $M \subseteq N \backslash\{i, j\}$. In particular, (5) holds for any $i, j \in N$ with $i \neq j$ and $M=N \backslash\{i, j\}$. For $M=N \backslash\{i, j\}$, the left side of (5) coincides with $\mathscr{L}_{i j}(\theta)$ and thus $\mathscr{L}_{i j}(\theta) \leq 0$ for all $i, j \in N$ with $i \neq j$.

$(\Leftarrow)$ Now, we assume that

$$
\mathscr{L}_{i j}(\theta) \leq 0
$$

for all $i, j \in N$ with $i \neq j$. Then, for a given $i, j \in N$ with $i \neq j$ it holds that

$$
A_{j}\left(\sum_{k \in N} p_{k} A_{i}-p_{i}\right)-p_{j} A_{i} \leq 0
$$

Now, let $M \subseteq N \backslash\{i, j\}$. As $\sum_{k \in M \cup\{i, j\}} p_{k} A_{i} \leq \sum_{k \in N} p_{k} A_{i}$, we can conclude that

$$
A_{j}\left(\sum_{k \in M \cup\{i, j\}} p_{k} A_{i}-p_{i}\right)-p_{j} A_{i} \leq A_{j}\left(\sum_{k \in N} p_{k} A_{i}-p_{i}\right)-p_{j} A_{i} \leq 0 .
$$


This implies that

$$
\begin{aligned}
0 \leq & -A_{j}\left(\sum_{k \in M \cup\{i, j\}} p_{k} A_{i}-p_{i}\right)+p_{j} A_{i} \\
= & -A_{j}\left(\sum_{k \in M \cup\{i, j\}} p_{k} A_{i}-p_{i}\right) \\
& +\left(\sum_{k \in M \cup\{i, j\}} p_{k} A_{i}-p_{i}\right)-\left(\sum_{k \in M \cup\{i\}} p_{k} A_{i}-p_{i}\right) \\
= & \left(1-A_{j}\right)\left(\sum_{k \in M \cup\{i, j\}} p_{k} A_{i}-p_{i}\right)-\left(\sum_{k \in M \cup\{i\}} p_{k} A_{i}-p_{i}\right)
\end{aligned}
$$

Multiplying the last expression by $\prod_{l \in M}\left(1-A_{l}\right)>0$ results in

$$
\prod_{l \in M}\left(1-A_{l}\right)\left(\left(1-A_{j}\right)\left(\sum_{k \in M \cup\{i, j\}} p_{k} A_{i}-p_{i}\right)-\left(\sum_{k \in M \cup\{i\}} p_{k} A_{i}-p_{i}\right)\right) \geq 0 .
$$

From proof $(\Rightarrow)$, we know that this inequality coincides with

$$
v(M \cup\{i, j\})-v(M \cup\{j\})-(v(M \cup\{i\})-v(M)) \geq 0 .
$$

As $i, j \in N$ with $i \neq j$ and $M \subseteq N \backslash\{i, j\}$ were chosen arbitrarily, we can conclude that (6) holds for any $i, j \in N$ with $i \neq j$ and all $M \subseteq N \backslash\{i, j\}$. Using recursive arguments, it can be seen that (6) is sufficient to show convexity.

Proof of Corollary 2 Let $\theta \in \Theta^{L}$ be a linear availability situation with $N=$ $\{1,2, \ldots, n\}$ with $n \geq 2, p_{i}=\bar{p} \in \mathbb{R}_{+}$for all $i \in N$ and $A_{1} \geq A_{2} \geq \cdots \geq A_{n}$. Let $\left(N, v^{\theta}\right)$ be the corresponding availability game. We will show that the corresponding availability game is convex if and only if $|N| A_{1} A_{2}-A_{1}-A_{2} \leq 0$.

$(\Rightarrow)$ Suppose the corresponding availability game is convex. Then, by Theorem 3 , $\mathscr{L}_{i j}(\theta) \leq 0$ for all $i, j \in N$ with $i \neq j$ and so

$$
\mathscr{L}_{12}(\theta)=A_{2}\left(|N| \bar{p} A_{1}-\bar{p}\right)-\bar{p} A_{1} \leq 0 .
$$

As $\bar{p} \in \mathbb{R}_{+}$, we derive

$$
|N| A_{1} A_{2}-A_{1}-A_{2} \leq 0,
$$

which concludes this part of the proof.

$(\Leftarrow)$ Suppose that $|N| A_{1} A_{2}-A_{1}-A_{2} \leq 0$. Then, it holds that 


$$
A_{1}\left(\frac{1}{2}|N| A_{2}-1\right)+A_{2}\left(\frac{1}{2}|N| A_{1}-1\right) \leq 0 .
$$

As $0 \leq A_{2} \leq A_{1}<1$, this implies that $\frac{1}{2}|N| A_{2}-1 \leq 0$ and $\frac{1}{2}|N| A_{1}-1 \leq 0$ or $\frac{1}{2}|N| A_{2}-1 \leq 0$ and $\frac{1}{2}|N| A_{1}-1 \geq 0$. We will now investigate those different cases.

Case 1. $\frac{1}{2}|N| A_{2}-1 \leq 0$ and $\frac{1}{2}|N| A_{1}-1 \leq 0$.

As $\frac{1}{2}|N| A_{1}-1 \leq 0$, it holds that $\frac{1}{2}|N| A_{j}-1 \leq \frac{1}{2}|N| A_{1}-1 \leq 0$ for all $j \in N$. As $A_{i} \in(0,1)$ for all $i \in N$, it holds that $A_{i}\left(\frac{1}{2}|N| A_{j}-1\right) \leq 0$ for all $i, j \in N$. So, for all $i, j \in N$ with $i \neq j$, it holds that

$$
A_{i}\left(\frac{1}{2}|N| A_{j}-1\right)+A_{j}\left(\frac{1}{2}|N| A_{i}-1\right) \leq 0 .
$$

Case 2. $\frac{1}{2}|N| A_{2}-1 \leq 0$ and $\frac{1}{2}|N| A_{1}-1 \geq 0$.

As $A_{1}\left(\frac{1}{2}|N| A_{j}-1\right) \leq A_{1}\left(\frac{1}{2}|N| A_{2}-1\right)$ for all $j \in N \backslash\{1\}$ and $A_{j}\left(\frac{1}{2}|N| A_{1}-\right.$ 1) $\leq A_{2}\left(\frac{1}{2}|N| A_{1}-1\right)$ for all $j \in N \backslash\{1\}$, it holds that

$$
\begin{aligned}
A_{1}\left(\frac{1}{2}|N| A_{j}-1\right)+A_{j}\left(\frac{1}{2}|N| A_{1}-1\right) \leq & A_{1}\left(\frac{1}{2}|N| A_{2}-1\right) \\
& +A_{2}\left(\frac{1}{2}|N| A_{1}-1\right) \leq 0
\end{aligned}
$$

for all $j \in N \backslash\{1\}$. For $i \in N \backslash\{1\}$ and $j \in N: j>i$, it holds that $\frac{1}{2}|N| A_{j}-$ $1 \leq \frac{1}{2}|N| A_{i}-1 \leq \frac{1}{2}|N| A_{2}-1 \leq 0$. Thus

$$
A_{i}\left(\frac{1}{2}|N| A_{j}-1\right)+A_{j}\left(\frac{1}{2}|N| A_{i}-1\right) \leq 0 .
$$

Combining Case 1 and Case 2, we conclude that (8) holds for all $i \in N$ and all $j \in N$ with $j>i$. Since $\mathscr{L}_{i j}(\theta)=\mathscr{L}_{j i}(\theta)$ for all $i, j \in N$ with $i \neq j$, (8) holds for all $i, j \in N$ with $i \neq j$. As multiplying (8) with $\bar{p} \in \mathbb{R}_{+}$will not affect the right-hand side of the inequality, it holds that $\mathscr{L}_{i j}(\theta) \leq 0$ for all $i, j \in N$ with $i \neq j$. By Theorem 3 , the corresponding availability game is convex.

Proof of Corollary 3 Let $\theta \in \Theta^{L}$ be a linear availability situation with $N=$ $\{1,2, \ldots, n\}$ with $n \geq 2, A_{i}=\bar{A} \in(0,1)$ for all $i \in N$ and $p_{1} \leq p_{2} \cdots \leq p_{n}$. Let $\left(N, v^{\theta}\right)$ be the corresponding availability game. We will show that the corresponding availability game is convex if and only if $\bar{A} \leq \frac{p_{1}+p_{2}}{\sum_{i \in N} p_{i}}$.

$(\Rightarrow)$ Suppose the corresponding availability game is convex. Then, by Theorem 3 , $\mathscr{L}_{i j}(\theta) \leq 0$ for all $i, j \in N$ with $i \neq j$ and so

$$
\mathscr{L}_{12}(\theta)=\bar{A}\left(\sum_{i \in N} p_{i} \bar{A}-p_{1}\right)-p_{2} \bar{A} \leq 0 .
$$


After some rewriting, we derive

$$
\bar{A} \leq \frac{p_{1}+p_{2}}{\sum_{i \in N} p_{i}}
$$

which concludes this part of the proof.

$(\Leftarrow)$ Suppose that $0<\bar{A} \leq \frac{p_{1}+p_{2}}{\sum_{i \in N} p_{i}}$. After some rewriting, we derive

$$
\bar{A}\left(\sum_{i \in N} p_{i} \bar{A}-p_{1}\right)-p_{2} \bar{A} \leq 0
$$

The left-hand side of (9) coincides with $\mathscr{L}_{12}(\theta)$, and so $\mathscr{L}_{12}(\theta) \leq 0$. Now, observe that

$$
\begin{aligned}
0 \geq \mathscr{L}_{12}(\theta)=\bar{A}\left(\sum_{k \in N} p_{k} \bar{A}-p_{1}\right)-p_{2} \bar{A} & =\bar{A}^{2} \sum_{k \in N} p_{k}-\bar{A}\left(p_{1}+p_{2}\right) \\
& \geq \bar{A}^{2} \sum_{k \in N} p_{k}-\bar{A}\left(p_{i}+p_{j}\right) \\
& =\mathscr{L}_{i j}(\theta)
\end{aligned}
$$

for all $i, j \in N$ with $i \neq j$. This implies that $\mathscr{L}_{i j}(\theta) \leq 0$ for all $i, j \in N$ with $i \neq j$. By Theorem 3 , the corresponding availability game is convex.

Proof of Corollary 4 Let $\theta \in \Theta^{L}$ be a linear availability situation with $N=$ $\{1,2, \ldots, n\}$ with $n \geq 2, A_{i}=\bar{A} \in(0,1)$ for all $i \in N$ and $p_{i}=\bar{p} \in \mathbb{R}_{+}$for all $i \in N$. Let $\left(N, v^{\theta}\right)$ be the corresponding availability game. We will show that the corresponding availability game is convex if and only if $\bar{A} \leq \frac{2}{|N|}$.

$(\Rightarrow)$ Suppose the corresponding availability game is convex. Then, by Corollary 3 , it holds that

$$
\bar{A} \leq \frac{p_{1}+p_{2}}{\sum_{k \in N} p_{k}}=\frac{2 \bar{p}}{|N| \bar{p}}=\frac{2}{|N|},
$$

which concludes the first part of the proof.

$(\Leftarrow)$ Suppose that $\bar{A} \leq \frac{2}{|N|}$. This implies that

$$
\bar{A} \leq \frac{2}{|N|}=\frac{2 \bar{p}}{|N| \bar{p}}=\frac{p_{1}+p_{2}}{\sum_{k \in N} p_{k}}
$$

and thus, by Corollary 3, the corresponding game is convex.

Proof of Proposition 3 Let $\theta \in \Theta$ be an availability situation and $\theta^{\prime} \in \Theta$ be another availability situation that coincides with $\theta$ except for the availability of player $j$, i.e. $A_{j} \leq A_{j}^{\prime}$. Then, it holds for any player $i \in N$ that 


$$
\begin{aligned}
\mathrm{OP}_{i}(\theta) & =P_{i}\left(1-\prod_{k \in N}\left(1-A_{k}\right)\right) \\
& =P_{i}\left(1-\prod_{k \in N \backslash\{j\}}\left(1-A_{k}\right)\left(1-A_{j}\right)\right) \\
& =P_{i}^{\prime}\left(1-\prod_{k \in N \backslash\{j\}}\left(1-A_{k}^{\prime}\right)\left(1-A_{j}\right)\right) \\
& \leq P_{i}^{\prime}\left(1-\prod_{k \in N \backslash\{j\}}\left(1-A_{k}^{\prime}\right)\left(1-A_{j}^{\prime}\right)\right) \\
& =\mathrm{OP}_{i}\left(\theta^{\prime}\right) .
\end{aligned}
$$

where the third equality results from $A_{k}=A_{k}^{\prime}$ for all $k \neq j$ and $P_{i}=P_{i}^{\prime}$ for all $i \in N$. The inequality results from $0 \leq A_{j} \leq A_{j}^{\prime} \leq 1$ with $0 \leq \prod_{k \in N \backslash\{j\}}\left(1-A_{k}^{\prime}\right) \leq 1$, and the fact that $P_{i}$ is non-decreasing.

Proof of Proposition 4 (i) OP From Proposition 3, it follows that allocation rule OP satisfies monotonicity to availability on $\Theta$. As $\Theta^{L} \subseteq \Theta$, allocation rule OP satisfies monotonicity to availability on $\Theta^{L}$ as well.

(ii) SPP Let $\theta \in \Theta^{L}$ be a linear availability situation and $\theta^{\prime} \in \Theta^{L}$ be another linear availability situation that only deviates in the availability of player $j \in N$ with $A_{j} \leq$ $A_{j}^{\prime}$. We claim that the derivative of $\operatorname{SPP}_{i}(\theta)$ for any player $i \in N$ is non-negative with respect to availability $A_{j}$. Note that $P_{i}(1)-P_{i}\left(A_{i}\right)=p_{i}-p_{i} A_{i}=p_{i}\left(1-A_{i}\right)$ for all $i \in N$.

Allocation $\operatorname{SPP}_{i}(\theta)$ for player $i \in N$ can be rewritten as

$$
\begin{aligned}
\operatorname{SPP}_{i}(\theta)= & p_{i} A_{i}+\frac{p_{i}\left(1-A_{i}\right)}{\sum_{k \in N} p_{k}\left(1-A_{k}\right)}\left(\sum_{t \in N} p_{t}\left(1-\prod_{k \in N}\left(1-A_{k}\right)\right)-\sum_{l \in N} p_{l} A_{l}\right) \\
= & p_{i} A_{i}+\left(1-\frac{\sum_{l \in N \backslash\{i\}} p_{l}\left(1-A_{l}\right)}{\sum_{l \in N} p_{l}\left(1-A_{l}\right)}\right) \times \\
& \left(\sum_{t \in N} p_{t}\left(1-A_{t}-\prod_{k \in N}\left(1-A_{k}\right)\right)\right) \\
= & p_{i} A_{i}+\sum_{t \in N} p_{t}\left(1-A_{t}-\prod_{k \in N}\left(1-A_{k}\right)\right)-\sum_{l \in N \backslash\{i\}} p_{l}\left(1-A_{l}\right) \\
& +\frac{\sum_{l \in N \backslash\{i\}} p_{l}\left(1-A_{l}\right)}{\sum_{l \in N} p_{l}\left(1-A_{l}\right)} \sum_{t \in N} p_{t} \prod_{k \in N}\left(1-A_{k}\right)
\end{aligned}
$$




$$
\begin{aligned}
= & p_{i} A_{i}+p_{i}\left(1-A_{i}\right)-\sum_{t \in N} p_{t} \prod_{k \in N}\left(1-A_{k}\right) \\
& +\frac{\sum_{l \in N \backslash\{i\}} p_{l}\left(1-A_{l}\right)}{\sum_{l \in N} p_{l}\left(1-A_{l}\right)} \sum_{t \in N} p_{t} \prod_{k \in N}\left(1-A_{k}\right) \\
= & p_{i}-\sum_{t \in N} p_{t} \prod_{k \in N}\left(1-A_{k}\right)\left(1-\frac{\sum_{l \in N \backslash\{i\}} p_{l}\left(1-A_{l}\right)}{\sum_{l \in N} p_{l}\left(1-A_{l}\right)}\right) \\
= & p_{i}-\sum_{t \in N} p_{t} \prod_{k \in N}\left(1-A_{k}\right)\left(\frac{p_{i}\left(1-A_{i}\right)}{\sum_{l \in N} p_{l}\left(1-A_{l}\right)}\right) .
\end{aligned}
$$

As at least for one player $k \in N, p_{k}(1)-p_{k}\left(A_{k}\right)>0$, function $\operatorname{SPP}_{i}(\theta)$ is continuous and differentiable in $A_{j}$. For player $j$, the derivative of $\operatorname{SPP}_{j}(\theta)$ to $A_{j}$ is given by

$$
\begin{aligned}
\frac{\mathrm{d}}{\mathrm{d} A_{j}} \operatorname{SPP}_{j}(\theta)= & -\frac{\sum_{l \in N} p_{l}\left(1-A_{l}\right) \cdot\left(-p_{j}\right)-p_{j}\left(1-A_{j}\right) \cdot\left(-p_{j}\right)}{\left(\sum_{l \in N} p_{l}\left(1-A_{l}\right)\right)^{2}} \sum_{t \in N} p_{t} \prod_{k \in N}\left(1-A_{k}\right) \\
& -\frac{p_{j}\left(1-A_{j}\right)}{\sum_{l \in N} p_{l}\left(1-A_{l}\right)} \sum_{t \in N} p_{t} \prod_{k \in N \backslash\{j\}}\left(1-A_{k}\right) \cdot(-1) \\
= & \frac{p_{j}}{\left(\sum_{l \in N} p_{l}\left(1-A_{l}\right)\right)^{2}}\left(\sum_{l \in N \backslash\{j\}} p_{l}\left(1-A_{l}\right) \sum_{t \in N} p_{t} \prod_{k \in N}\left(1-A_{k}\right)\right. \\
& \left.+\sum_{l \in N} p_{l}\left(1-A_{l}\right) \sum_{t \in N} p_{t} \prod_{k \in N}\left(1-A_{k}\right)\right) \\
= & \frac{p_{j} \sum_{t \in N} p_{t} \prod_{k \in N}\left(1-A_{k}\right)}{\left(\sum_{l \in N} p_{l}\left(1-A_{l}\right)\right)^{2}}\left(\sum_{l \in N \backslash\{j\}} p_{l}\left(1-A_{l}\right)+\sum_{l \in N} p_{l}\left(1-A_{l}\right)\right) \geq 0 .
\end{aligned}
$$

Note that all terms are non-negative, and thus, the derivative is non-negative as well. Hence, $\operatorname{SPP}_{j}(\theta)$ is non-decreasing in $A_{j}$. This implies that $\operatorname{SPP}_{j}(\theta) \leq \operatorname{SPP}_{j}\left(\theta^{\prime}\right)$. Taking the derivative of $\operatorname{SPP}_{i}(\theta)$ to $A_{j}$ with $i \in N \backslash\{j\}$ gives

$$
\begin{aligned}
\frac{\mathrm{d}}{\mathrm{d} A_{j}} \operatorname{SPP}_{i}(\theta)= & -\frac{0-\left(p_{i}\left(1-A_{i}\right) \cdot\left(-p_{j}\right)\right)}{\left(\sum_{l \in N} p_{l}\left(1-A_{l}\right)\right)^{2}} \sum_{t \in N} p_{t} \prod_{k \in N}\left(1-A_{k}\right) \\
& -\frac{p_{i}\left(1-A_{i}\right)}{\sum_{l \in N} p_{l}\left(1-A_{l}\right)} \sum_{t \in N} p_{t} \prod_{k \in N \backslash\{j\}}\left(1-A_{k}\right) \cdot(-1) \\
= & \frac{p_{i}\left(1-A_{i}\right)}{\left(\sum_{l \in N} p_{l}\left(1-A_{l}\right)\right)^{2}}\left(-p_{j} \sum_{t \in N} p_{t} \prod_{k \in N}\left(1-A_{k}\right)\right. \\
& \left.+\sum_{t \in N} p_{t} \prod_{k \in N \backslash\{j\}}\left(1-A_{k}\right) \sum_{l \in N} p_{l}\left(1-A_{l}\right)\right)
\end{aligned}
$$




$$
\begin{aligned}
= & \frac{p_{i}\left(1-A_{i}\right)}{\left(\sum_{l \in N} p_{l}\left(1-A_{l}\right)\right)^{2}}\left(\sum_{t \in N} p_{t} \prod_{k \in N \backslash\{j\}}\left(1-A_{k}\right)\right) \\
& \times\left(-p_{j}\left(1-A_{j}\right)+\sum_{l \in N} p_{l}\left(1-A_{l}\right)\right) \\
= & \frac{p_{i}\left(1-A_{i}\right)}{\left(\sum_{l \in N} p_{l}\left(1-A_{l}\right)\right)^{2}}\left(\sum_{t \in N} p_{t} \prod_{k \in N \backslash\{j\}}\left(1-A_{k}\right)\right) \\
& \times\left(\sum_{l \in N \backslash\{j\}} p_{l}\left(1-A_{l}\right)\right) \geq 0 .
\end{aligned}
$$

Note that all terms are non-negative, and thus, the derivative is non-negative as well. Hence, $\operatorname{SPP}_{i}(\theta)$ is non-decreasing in $A_{j}$ for all $i \in N \backslash\{j\}$. We conclude that $\operatorname{SPP}_{i}(\theta)$ $\leq \operatorname{SPP}_{i}\left(\theta^{\prime}\right)$ for all $i \in N$.

Proof of Proposition 5 Let $\theta, \theta^{\prime} \in \Theta$ be two availability situations where $\theta$ and $\theta^{\prime}$ coincide except for the profit of player $j$ with $P_{j}^{\prime}(x)-P_{j}(x)$ non-decreasing in $x$. As $P_{k}^{\prime}(x)-P_{k}(x)=0$ for all $k \in N \backslash\{j\}$, it holds that $P_{i}^{\prime}(x) \geq P_{i}(x)$ for all $i \in N$. Hence, it holds for all $i \in N$ that

$$
\begin{aligned}
\mathrm{OP}_{i}(\theta) & =P_{i}\left(1-\prod_{k \in N}\left(1-A_{k}\right)\right) \\
& \leq P_{i}^{\prime}\left(1-\prod_{k \in N}\left(1-A_{k}^{\prime}\right)\right)=\mathrm{OP}_{i}\left(\theta^{\prime}\right)
\end{aligned}
$$

given that $A_{k}=A_{k}^{\prime}$ for all $k \in N$.

In the same line, it holds that

$$
\begin{aligned}
\mathrm{PA}_{i}(\theta) & =\frac{A_{i}}{\sum_{k \in N} A_{k}} \sum_{k \in N} P_{k}\left(1-\prod_{h \in N}\left(1-A_{h}\right)\right) \\
& \leq \frac{A_{i}^{\prime}}{\sum_{k \in N} A_{k}^{\prime}} \sum_{k \in N} P_{k}^{\prime}\left(1-\prod_{h \in N}\left(1-A_{h}^{\prime}\right)\right)=\mathrm{PA}_{i}\left(\theta^{\prime}\right),
\end{aligned}
$$

given that $A_{k}=A_{k}^{\prime}$ for all $k \in N$.

Finally, let $i \in N$ and $M \subseteq N \backslash\{i\}$. Then

$$
\begin{aligned}
v^{\theta^{\prime}}(M \cup\{i\})-v^{\theta^{\prime}}(M)= & \sum_{k \in M \cup\{i\}} P_{k}^{\prime}\left(1-\prod_{l \in M \cup\{i\}}\left(1-A_{l}\right)\right) \\
& -\sum_{k \in M} P_{k}^{\prime}\left(1-\prod_{l \in M}\left(1-A_{l}\right)\right)
\end{aligned}
$$




$$
\begin{aligned}
= & \sum_{k \in M}\left(P_{k}^{\prime}\left(1-\prod_{l \in M \cup\{i\}}\left(1-A_{l}\right)\right)\right. \\
& \left.-P_{k}^{\prime}\left(1-\prod_{l \in M}\left(1-A_{l}\right)\right)\right)+P_{i}^{\prime}\left(1-\prod_{l \in M \cup\{i\}}\left(1-A_{l}\right)\right) \\
\geq & \sum_{k \in M}\left(P_{k}\left(1-\prod_{l \in M \cup\{i\}}\left(1-A_{l}\right)\right)\right. \\
& \left.-P_{k}\left(1-\prod_{l \in M}\left(1-A_{l}\right)\right)\right)+P_{i}\left(1-\prod_{l \in M \cup\{i\}}\left(1-A_{l}\right)\right) \\
= & v^{\theta}(M \cup\{i\})-v^{\theta}(M),
\end{aligned}
$$

where the inequality holds, as

(i) if $j \in M$ (and thus $j \neq i$ ) then $P_{j}^{\prime}(y)-P_{j}^{\prime}(x) \geq P_{j}(y)-P_{j}(x)$ for $y \geq x$ and $P_{l}^{\prime}=P_{l}$ for all $l \in M \backslash\{j\}$ and $P_{i}^{\prime}=P_{i}$.

(ii) if $j \notin M$ and $i=j$ then $P_{l}^{\prime}=P_{l}$ for all $l \in M$ and $P_{i}^{\prime} \geq P_{i}$.

(iii) if $j \notin M$ and $i \neq j$ then $P_{l}^{\prime}=P_{l}$ for all $l \in M$ and $P_{i}^{\prime}=P_{i}$ and so the inequality becomes equality.

As $v^{\theta^{\prime}}(M \cup\{i\})-v^{\theta^{\prime}}(M) \geq v^{\theta}(M \cup\{i\})-v^{\theta}(M)$ for any $i \in N$ and $M \subseteq N \backslash\{i\}$ and given that the Shapley value of a cooperative game is a weighted average over those marginal contributions, it follows that

$$
\mathrm{SV}_{i}(\theta)=\Phi_{i}\left(N, v^{\theta}\right) \leq \Phi_{i}\left(N, v^{\theta^{\prime}}\right)=\mathrm{SV}_{i}\left(\theta^{\prime}\right) \text { for all } i \in N
$$

which concludes the proof.

Proof of Proposition 6 Let $\theta \in \Theta$ be an availability situation and $i, j \in N$ with $i \neq j$ two players that are situation symmetric. For allocation rule OP, it holds that

$$
\mathrm{OP}_{i}(\theta)=P_{i}\left(1-\prod_{k \in N}\left(1-A_{k}\right)\right)=P_{j}\left(1-\prod_{k \in N}\left(1-A_{k}\right)\right)=\mathrm{OP}_{j}(\theta)
$$

as $P_{i}(x)=P_{j}(x)$ for all $x \in[0,1]$. For allocation rule PA, it holds that

$$
\mathrm{PA}_{i}(\theta)=\frac{A_{i}}{\sum_{k \in N} A_{k}} v^{\theta}(N)=\frac{A_{j}}{\sum_{k \in N} A_{k}} v^{\theta}(N)=\mathrm{PA}_{j}(\theta)
$$


as $A_{i}=A_{j}$. For allocation rule SPP, it holds that

$$
\begin{aligned}
\operatorname{SPP}_{i}(\theta) & =v^{\theta}(\{i\})+\frac{P_{i}(1)-P_{i}\left(A_{i}\right)}{\sum_{k \in N}\left[P_{k}(1)-P_{k}\left(A_{k}\right)\right]}\left(v^{\theta}(N)-\sum_{l \in N} v^{\theta}(\{l\})\right) \\
& =P_{i}\left(A_{i}\right)+\frac{P_{i}(1)-P_{i}\left(A_{i}\right)}{\sum_{k \in N}\left[P_{k}(1)-P_{k}\left(A_{k}\right)\right]}\left(v^{\theta}(N)-\sum_{l \in N} v^{\theta}(\{l\})\right) \\
& =P_{j}\left(A_{j}\right)+\frac{P_{j}(1)-P_{j}\left(A_{j}\right)}{\sum_{k \in N}\left[P_{k}(1)-P_{k}\left(A_{k}\right)\right]}\left(v^{\theta}(N)-\sum_{l \in N} v^{\theta}(\{l\})\right) \\
& =v^{\theta}(\{j\})+\frac{P_{j}(1)-P_{j}\left(A_{j}\right)}{\sum_{k \in N}\left[P_{k}(1)-P_{k}\left(A_{k}\right)\right]}\left(v^{\theta}(N)-\sum_{l \in N} v^{\theta}(\{l\})\right) \\
& =\operatorname{SPP}_{j}(\theta),
\end{aligned}
$$

As $P_{i}(x)=P_{j}(x)$ for all $x \in[0,1]$ and $A_{i}=A_{j}$. Finally, for allocation rule $\mathrm{SV}$, it holds that $P_{i}(x)=P_{j}(x)$ for all $x \in[0,1]$ and $A_{i}=A_{j}$. This implies that $v^{\theta}(M \cup\{i\})=v^{\theta}(M \cup\{j\})$ for all $M \subseteq N \backslash\{i, j\}$. Based on Definition 8, players $i$ and $j$ are game symmetric. Based on Proposition 7 and Definition 9, we conclude that $\mathrm{SV}_{i}(\theta)=\mathrm{SV}_{j}(\theta)$.

Proof of Lemma 3 Let $\theta \in \Theta^{L}$ be a linear availability situation, $x_{i}=1-A_{i}$ for all $i \in N$, and $M \subseteq N$. Then, it holds that

$$
\sum_{i \in M} p_{i} x_{i} \sum_{j \in M} p_{j}\left(1-\prod_{k \in N \backslash M} x_{k}\right) \geq 0
$$

Moreover, it holds that

$$
\sum_{i \in N \backslash M} p_{i} x_{i} \sum_{k \in M} p_{k}-\sum_{i \in N \backslash M} p_{i} \prod_{j \in N \backslash M} x_{j} \sum_{k \in M} p_{k} x_{k} \geq 0 .
$$

Now, when both parts are summed, we obtain

$$
\begin{aligned}
0 \leq & \sum_{i \in M} p_{i} x_{i} \sum_{j \in M} p_{j}\left(1-\prod_{k \in N \backslash M} x_{k}\right)+\sum_{i \in N \backslash M} p_{i} x_{i} \sum_{k \in M} p_{k} \\
& -\sum_{i \in N \backslash M} p_{i} \prod_{j \in N \backslash M} x_{j} \sum_{k \in M} p_{k} x_{k} \\
= & \sum_{i \in M} p_{i} x_{i} \sum_{j \in M} p_{j}-\sum_{i \in M} p_{i} x_{i} \sum_{j \in M} p_{j} \prod_{k \in N \backslash M} x_{k}+\sum_{i \in N \backslash M} p_{i} x_{i} \sum_{k \in M} p_{k} \\
& -\sum_{i \in N \backslash M} p_{i} \prod_{j \in N \backslash M} x_{j} \sum_{k \in M} p_{k} x_{k}
\end{aligned}
$$




$$
\begin{aligned}
& =\sum_{i \in N} p_{i} x_{i} \sum_{j \in M} p_{j}-\sum_{i \in M} p_{i} x_{i} \sum_{j \in M} p_{j} \prod_{k \in N \backslash M} x_{k}-\sum_{i \in N \backslash M} p_{i} \prod_{j \in N \backslash M} x_{j} \sum_{k \in M} p_{k} x_{k} \\
& =\sum_{i \in N} p_{i} x_{i} \sum_{j \in M} p_{j}-\sum_{i \in M} p_{i} x_{i} \sum_{j \in M} p_{j} \prod_{k \in N \backslash M} x_{k}-\sum_{k \in M} p_{k} x_{k} \sum_{i \in N \backslash M} p_{i}\left(\prod_{j \in N \backslash M} x_{j}\right) \\
& =\sum_{i \in N} p_{i} x_{i} \sum_{j \in M} p_{j}-\sum_{i \in M} p_{i} x_{i} \sum_{j \in N} p_{j}\left(\prod_{k \in N \backslash M} x_{k}\right)
\end{aligned}
$$

where the equalities hold by rewriting. From the last expression, we derive

$$
\sum_{i \in N} p_{i} x_{i} \sum_{j \in M} p_{j} \geq \sum_{i \in M} p_{i} x_{i} \sum_{j \in N} p_{j}\left(\prod_{k \in N \backslash M} x_{k}\right)
$$

Multiplying both sides by $\prod_{j \in M} x_{j}(\geq 0)$ and subsequently dividing both sides by $\sum_{j \in N} p_{j} x_{j}(>0)$ gives

$$
\sum_{i \in M} p_{i} \prod_{j \in M} x_{i} \geq \frac{\sum_{i \in M} p_{i} x_{i}}{\sum_{j \in N} p_{j} x_{j}} \sum_{i \in N} p_{i} \prod_{j \in N} x_{i}
$$

which concludes the proof.

Proof of Proposition 8 Let $\theta \in \Theta^{L}$ be a linear availability situation. Note that $P_{i}(1)-$ $P_{i}\left(A_{i}\right)=p_{i}-p_{i} A_{i}=p_{i}\left(1-A_{i}\right)$ for all $i \in N$. It holds that

$$
\begin{aligned}
\sum_{i \in N} \operatorname{SPP}_{i}(\theta) & =\sum_{i \in N}\left(v^{\theta}(\{i\})+\frac{p_{i}\left(1-A_{i}\right)}{\sum_{j \in N} p_{j}\left(1-A_{j}\right)}\left(v^{\theta}(N)-\sum_{k \in N} v^{\theta}(\{k\})\right)\right) \\
& =\sum_{i \in N} v^{\theta}(\{i\})+v^{\theta}(N)-\sum_{k \in N} v^{\theta}(\{k\}) \\
& =v^{\theta}(N),
\end{aligned}
$$

and thus, $\operatorname{SPP}(\theta)$ is efficient. Secondly, let $M \subseteq N$, then

$$
\begin{aligned}
v^{\theta}(M) & =\sum_{i \in M} p_{i}\left(1-\prod_{j \in M}\left(1-A_{j}\right)\right) \\
& =\sum_{i \in M} p_{i} A_{i}+\sum_{i \in M} p_{i}\left(1-A_{i}\right)-\sum_{i \in M} p_{i} \prod_{j \in M}\left(1-A_{j}\right) \\
& \leq \sum_{i \in M} v^{\theta}(\{i\})+\sum_{i \in M} p_{i}\left(1-A_{i}\right)-\frac{\sum_{i \in M} p_{i}\left(1-A_{i}\right)}{\sum_{k \in N} p_{k}\left(1-A_{k}\right)} \sum_{i \in N} p_{i} \prod_{j \in N}\left(1-A_{j}\right)
\end{aligned}
$$




$$
\begin{aligned}
& =\sum_{i \in M} v^{\theta}(\{i\})+\frac{\sum_{i \in M} p_{i}\left(1-A_{i}\right)}{\sum_{k \in N} p_{k}\left(1-A_{k}\right)}\left(\sum_{k \in N} p_{k}\left(1-A_{k}\right)-\sum_{i \in N} p_{i} \prod_{j \in N}\left(1-A_{j}\right)\right) \\
& =\sum_{i \in M} v^{\theta}(\{i\})+\frac{\sum_{i \in M} p_{i}\left(1-A_{i}\right)}{\sum_{k \in N} p_{k}\left(1-A_{k}\right)}\left(\sum_{k \in N} p_{k}\left(1-\prod_{j \in N}\left(1-A_{j}\right)\right)-\sum_{k \in N} p_{k} A_{k}\right) \\
& =\sum_{i \in M}\left(v^{\theta}(\{i\})+\frac{p_{i}\left(1-A_{i}\right)}{\sum_{k \in N} p_{k}\left(1-A_{k}\right)}\left(v^{\theta}(N)-\sum_{k \in N} v^{\theta}(\{k\})\right)\right) \\
& =\sum_{i \in M} \operatorname{SPP}_{i}(\theta),
\end{aligned}
$$

where the inequality is a result of Lemma 3 with $x_{j}=1-A_{j}$ for all $j \in N$. Hence, $\operatorname{SPP}(\theta)$ is also stable and thus a member of the core.

\section{References}

Bachrach Y, Meir R, Feldman M, Tennenholtz M (2011) Solving cooperative reliability games. In: Cozman FG, Pfeffer A (eds) Uncertainty in artificial intelligence. AUAI Press, Corvallis, Oregon, pp 27-34

Bachrach Y, Kash I, Shah N (2012) Agent failures in totally balanced games and convex games. In: Goldberg PW (ed) Internet and network economics. WINE 2012. Lecture notes in computer science. Springer, Berlin, pp 15-29

Bachrach Y, Porat E, Rosenschein J (2013) Sharing rewards in cooperative connectivity games. J Artif Intell Res 47:281-311

Bondareva O (1963) Some applications of linear programming methods to the theory of cooperative games. Probl Kibern 10:119-139

Borm P, Hamers H, Hendrickx R (2001) Operations research games: a survey. Top 9(2):139-199

Drechsel J, Kimms A (2011) Cooperative lot sizing with transshipments and scarce capacities: solutions and fair cost allocations. Int J Prod Res 49(9):2643-2668

Hezarkhani B, Slikker M, Van Woensel T (2016) A competitive solution for cooperative truckload delivery. OR Spectr 38(1):51-80

Karsten F, Basten R (2014) Pooling of spare parts between multiple users: how to share the benefits? Eur J Oper Res 233(1):94-104

Karsten F, Slikker M, van Houtum G (2012) Inventory pooling games for expensive, low-demand spare parts. Nav Res Logist 59(5):311-324

Karsten F, Slikker M, Van Houtum G (2015) Resource pooling and cost allocation among independent service providers. Oper Res 62(2):476-488

Li J, Cai X, Zeng Y (2016) Cost allocation for less-than-truckload collaboration among perishable product retailers. OR Spectr 38(1):81-117

Meca A, Timmer J, Garcia I, Borm P (2004) Inventory games. Eur J Oper Res 156(1):127-139

Muto S, Nakayama M, Potters J, Tijs S (1988) On big boss games. Econ Stud Q 39(4):303-321

Özen U, Fransoo J, Norde H, Slikker M (2008) Cooperation between multiple newsvendors with warehouses. Manuf Serv Oper Manag 10(2):311-324

Shapley L (1953) A value for n-person games. In: Tucker A, Kuhn H (eds) Contributions to the theory of games II. Princeton University Press, Princeton, pp 307-317

Shapley L (1967) On balanced sets and cores. Nav Res Logist Q 14(4):453-460

Sprumont Y (1990) Population monotonic allocation schemes for cooperative games with transferable utility. Games Econ Behav 2(4):378-394

Van den Heuvel W, Borm P, Hamers H (2007) Economic lot-sizing games. Eur J Oper Res 176(2):1117-1130 